\title{
XXVIII Olimpíadas, Atenas. O cardiologista está preparado?
}

\author{
Renata Rodrigues Teixeira de Castro, Antonio Claudio Lucas da Nóbrega, \\ Eduardo Henrique De Rose \\ Rio de Janeiro, RJ
}

Os XXVIII Jogos Olímpicos iniciar-se-ão em 13 de agosto próximo. Os meses que antecedem grandes competições são caracterizados pela ansiedade e busca do melhor desempenho pelos atletas. Freqüentemente, médicos não especialistas em medicina do esporte, inclusive cardiologistas, são procurados por atletas por diferentes motivações, uma vez que atletas não estão livres de serem acometidos por diversos tipos de doenças. Neste editorial, mostraremos que o cardiologista, comumente, prescreve substâncias que podem gerar caso positivo de doping, com conseqüentes transtornos não só para 0 atleta, mas também para o próprio médico.

Casos de doping vêm sendo, infelizmente, cada vez mais frequentes no esporte brasileiro e mundial ${ }^{1}$. Visando unificar as politicas de controle antidoping, nacionalmente, dentre os diferentes esportes olímpicos e, internacionalmente, dentre os diferentes países, no ano de 2003 representantes de diversas nacionalidades, incluindo o Brasil, reuniram-se em Copenhague, Dinamarca, e assinaram a Declaração de Copenhague sobre doping no esporte². A essa ocasião, o movimento olímpico no seu todo e as autoridades públicas presentes concordaram em seguir as determinações do Código Mundial Antidoping 3 . 0 objetivo do código é manter 0 chamado "espírito esportivo", caracterizando a prática esportiva pela ética, honestidade e saúde.

0 Código Mundial Antidoping aplica-se não só a atletas, mas também ao "pessoal de apoio ao atleta". Obviamente, pessoas que fazem parte da equipe do atleta, incluindo o médico da equipe ou aquele que acompanha o atleta, não serão submetidos ao controle antidoping, mas poderão ser punidos em caso de violação de alguma das regras constantes no Código. A prescrição ou tentativa de administração de uma substância ou método proibido para qualquer atleta é considerada doping. Além disso, o médico que ajuda, encoraja, encoberta ou exerce cumplicidade em qualquer caso de doping poderá ser punido ${ }^{3}$.

Vale ressaltar que não há necessidade de comprovar a intenção de melhora do desempenho para que seja definido um caso de doping. Resumidamente, um caso de doping subentende a presença de uma substância proibida, de seus marcadores ou seus metabólitos em um espécime proveniente do corpo de um atleta (mais comumente urina).

Laboratório de Ciências do Exercício, Depto de Fisiologia e Farmacologia, Universidade Federal Fluminense, Pós-Graduação em Cardiologia, Universidade Federal Fluminense, Departamento médico, Comitê Olímpico Brasileiro

Endereço para Correspondências: Renata Rodrigues Teixeira de Castro - Av. Paranapuã, 1849/201 - Cocotá - Cep 21910-005

Rio de Janeiro - RJ - E-mail: rrcastro@cardiol.br
Apesar de ser responsabilidade do atleta impedir a ingestão ou administração de substâncias proibidas ao seu próprio corpo, muitos atletas desconhecem as atualizações anuais da lista de substâncias proibidas publicadas pela WADA. Mais especificamente, $62,5 \%$ dos atletas das equipes brasileiras de taekwondo e natação, que participaram dos VIII Jogos Sul-Americanos em 2002, acreditavam que médicos generalistas (sem especialização em medicina do esporte) não prescreveriam substâncias capazes de configurar caso de doping ${ }^{4}$ pelo total conhecimento sobre 0 assunto.

A responsabilidade do médico que prescreveu a substância proibida não exime 0 atleta de sua punição. Em 2000, durante os Jogos Olímpicos de Sidney, houve um caso de doping que chamou a atenção específica da comunidade médica internacional ${ }^{5}$. Uma ginasta romena realizou controle antidoping positivo para pseudoefedrina e alegou ter utilizado medicamento contra a gripe prescrito pelo médico da equipe. Apesar da atleta ter sido punida, tendo perdido a medalha de ouro conquistada durante o evento, 0 Comitê Olímpico Internacional optou por punir também o médico responsável pela prescrição, proibindo sua participação nos dois Jogos Olímpicos subseqüentes (Jogos Olímpicos de Inverno de Salt Lake City-2002 e Jogos Olímpicos de Atenas-2004).

Obviamente, a prescrição de substâncias proibidas sem indicação clínica consistente, caracterizando o objetivo de melhora do desempenho do atleta é passivel de punição pelo Conselho Regional de Medicina, uma vez que indica conduta antiética ${ }^{6}$. Mas também não são raros os casos positivos de doping em que o médico prescreveu substância de acordo com o conhecimento científico existente. Diuréticos e betabloqueadores representam as principais substâncias presentes no receituário cardiológico que são proibidas para uso por atletas, de acordo com as seguintes situações.

\section{Diuréticos}

Algumas substâncias configuram casos de doping apenas quando detectadas acima de determinada concentração. Diuréticos são eventualmente utilizados como agentes mascaradores, ou seja, atuam diluindo a urina do atleta, diminuindo a concentração de outra substância proibida utilizada (descaracterizando um caso positivo de doping ${ }^{7}$. Outro objetivo freqüente, apesar de também proibido, do uso de diuréticos nos esportes é a redução rápida do peso corporal de atletas, que participam de esportes com divisão de categorias por peso ou onde o baixo peso corporal seja condição importante na obtenção de resultados ${ }^{8}$ (tab. I).

Ao pensar em atletas é comum visualizar-se pessoas jovens e 


\begin{tabular}{|l|}
\hline \multicolumn{1}{|c|}{ Tabela I - Esportes onde uso de diuréticos não será admitido } \\
\hline Fisiculturismo \\
Boxe \\
Judô \\
Karatê \\
Halterofilismo \\
Canoagem (peso leve) \\
Esqui (saltos) \\
Taekwondo \\
Lutas greco-romana e livre \\
\hline
\end{tabular}

saudáveis, verdadeiros exemplos de resistência e saúde. Entretanto, vale ressaltar que esportes caracterizados por precisão não exigem capacidade aeróbica elevada e, muitas vezes, seus campeões são atletas em faixas etárias onde cardiopatias e hipertensão são doenças freqüentes. Diuréticos podem ser necessários para o tratamento desses atletas e, nesses casos, o cardiologista deverá saber como proceder. Podemos citar como exemplo o caso de um atleta brasileiro de vela que, em controle antidoping fora de competição, apresentou resultado positivo para diurético. 0 medicamento havia sido inadvertidamente prescrito por seu cardiologista para o tratamento de hipertensão arterial sistêmica. Atletas que participem dos esportes descritos na tabela I não podem, independente da justificativa, utilizar diuréticos, uma vez que tais substâncias têm o potencial de melhorar seu desempenho esportivo. Entretanto, quando o esporte praticado pelo atleta não é caracterizado por qualquer benefício decorrente do baixo peso corporal, o uso de diurético poderá ser admitidos. Os procedimentos para obtenção desta concessão serão descritos abaixo.

A presença de qualquer substância proibida (independente de sua concentração) na urina de atleta que dispõe de concessão para utilização de diuréticos caracterizará caso positivo passivel de punição ${ }^{8}$.

\section{Betabloqueadores}

A última década caracterizou-se pela sedimentação dos benefícios do uso de betabloqueadores na prática cardiológica. Além de ser uma das opções para o tratamento da hipertensão arterial sistêmica ${ }^{9}$, os betabloqueadores são obrigatórios no tratamento ambulatorial de pacientes com doença arterial coronariana ${ }^{10}$ ou com insuficiência cardíaca, salvo contra-indicações precisas ${ }^{11}$.

Entretanto, a obtenção de melhores resultados em alguns esportes pode ser conseguida pela combinação de calma e precisão. Tais características podem ser alcançadas, de forma antiética, após ingestão de medicamento betabloqueador, uma vez que causa bradicardia, além de redução do tremor e diminuição da ansiedade $^{12}$. Sendo assim, o uso de betabloqueadores é proibido para atletas que participem das competições constantes na tabela II. As Federações Internacionais de Tiro e Tiro com Arco proíbem uso de betabloqueadores mesmo fora do período de competições ${ }^{8}$.

\section{Medicamentos permitidos}

A maioria dos medicamentos prescritos pelo cardiologista em sua prática diária, é permitida e não causa qualquer prejuízo para 0 atleta caso venha a realisar exame antidoping. Mais especifi-
Tabela II - Esportes onde beta-bloqueadores são proibidos

$\begin{array}{ll}\text { Aeronáuticos } & \text { Motociclismo } \\ \text { Automobilismo } & \text { Nado sincronizado } \\ \text { Bilhar } & \text { Pentatlo } \\ \text { Bobsleigh } & \text { Saltos ornamentais } \\ \text { Boliche } & \text { Tiro } \\ \text { Bridge } & \text { Tiro com arco } \\ \text { Futebol } & \text { Xadrez } \\ \text { Ginástica } & \text { Lutas } \\ \text { latismo } & \end{array}$

queadores de receptores da angiotensina II, bloqueadores de canal de cálcio, antiarrítmicos e hipolipemiantes, não oferecem risco de casos positivos de doping. Entretanto, vale ressaltar que a lista de substâncias proibidas é atualizada pela Agência Mundial Antidoping em $1^{\circ}$ de janeiro de cada ano. Sendo assim, caso haja evidências de melhora de desempenho com o uso de qualquer uma destas substâncias elas poderão vir a ser incluídas nestas atualizações. 0 Comitê Olímpico Brasileiro vem publicando, anualmente, um manual para orientação dos atletas contendo, não só a lista atualizada de substâncias proibidas, mas também uma lista de medicamentos (nomes comerciais) que podem ser utilizados em diferentes situações clínicas ${ }^{13}$.

\section{Solicitação de concessão para uso de substância restrita}

Antes de prescrever medicamentos a um atleta que participe de competições oficiais sugerimos que 0 cardiologista verifique a lista atualizada de substâncias proibidas ${ }^{9}$. A permissão para uso de substâncias proibidas, como anabolizantes, estimulantes, narcóticos e hormônios peptídeos não será concedida sob qualquer justificativa. 0 uso de betabloqueadores e diuréticos por atletas praticantes dos esportes incluidos nas tabelas I e II também não será autorizado.

Entretanto, o uso justificado de insulina em pacientes diabéticos insulino-dependentes, beta-agonistas para pacientes asmáticos ou de diuréticos em atletas cujos esportes não constem da tabela 1 poderá ser solicitado. Para tanto, o médico que acompanha 0 atleta deverá redigir relatório e anexar ao formulário de concessão para uso terapêutico (que pode ser conseguido em www.cob.org.br) ${ }^{13}$. Este formulário deverá ser enviado à Confederação esportiva a qual o atleta é filiado, para que possa ser avaliado e posteriormente concedido o uso da substância solicitada. No caso de solicitação de concessão para uso de beta-agonistas por atletas com asma ou broncoconstricção induzida pelo exercício, prova de função respiratória, caracterizando distúrbio obstrutivo poderá ser solicitada.

A necessidade de maiores esclarecimentos a respeito da justificativa para uso da medicação, incluindo os resultados dos exames, será avaliada por uma comissão médica independente.

\section{Conclusão}

A prescrição de substâncias proibidas, mesmo com indicação clínica inquestionável, é passível de punição pelo Comitê Olímpico Internacional, indicando suspensão da participação do médico de suas atividades em Jogos Olímpicos, Continentais ou Re- 
gionais posteriores. Além desta punição, devemos considerar que um caso positivo de doping pode indicar suspensão do atleta por um período que varia de seis meses a dois anos ( $1^{\text {a }}$ condenação) até 0 afastamento definitivo (reincidência). Desta forma, casos de doping, mesmo sem objetivo de melhora do desempenho, poderão prejudicar a carreira do médico e do atleta, ou seja, do paciente.
Considerando o grande número de medicamentos proibidos, as peculiaridades do controle antidoping dos diferentes esportes e a confiança total da maioria dos atletas em seu médico, é aconselhável que o cardiologista consulte a lista atualizada de substâncias proibidas sempre que realizar prescrição para um atleta sujeito ao controle antidoping.

\section{Referências}

1. World Anti-Doping Agency. Annual Report, 2002. WADA, Montreal, 2002.

2. World Anti-Doping Agency. Copenhagem declaration on anti-doping in sport. www.wada-ama.org

3. World Anti-Doping Agency. World anti-doping code. WADA, Montreal, 2003.

4. Castro RRT, Ramalho SHR, De Rose EH, Nóbrega ACL. Conhecimento de atletas de esportes olímpicos sobre controle antidoping: o tempo de participação do esporte nos jogos olímpicos faz diferença? Rev Bras Med Esporte 2003; 9: S13.

5. Birchard K. Olympic committee bans doctor after doping case. Lancet 2000; 356:1171.

6. Conselho Federal de Medicina. Código de ética médica-Resolução CFM 1246/88. Governo Federal - Diário Oficial da União, 08 janeiro de 1988.

7. Wagner JC. Enhancement of athletic performance with drugs. An overview. Sports Med. 1991; 12: 250-65.

8. World Anti-Doping Code. The 2004 prohibited list International standard. www.wada-ama.org
9. Chobanian AV, Bakris GL, Black HR, et al. Seventh report of the Joint National Committee on Prevention, Detection, Evaluation, and Treatment of High Blood Pressure. Hypertension. 2003; 42: 1206-52.

10. Abrams J, Chatterjee K, Daley J, et al. ACC/AHA 2002 Guideline Update for the management of patients with chronic stable angina. J Am Coll Cardiol. 2003; 41: $159-68$

11. Guimarães Jl, Mesquita ET, Bocchi EA, et al. Revisão das II Diretrizes da Sociedade Brasileira de Cardiologia para o diagnóstico e tratamento da insuficiência cardíaca. Arq Bras Cardiol 2002; 79 (supl IV): 1-30.

12. Kruse P, Ladefoged J, Nielsen U, Paulev PE, Sorensen JP. Beta-blockade used in precision sports: effect on pistol shooting performance. J Appl Physiol. 1986; 61: 417-20.

13. De Rose EH, Feder MG, Bento RMA, Aquino Neto FR. Uso de Medicamentos no Esporte. Comitê Olímpico Brasileiro, Rio de Janeiro, 2004.

Citalor ${ }^{\circledR}$ (atorvastatina cálcica) é um agente hipolipemiante que diminui os níveis plasmáticos de colesterol e lipoproteínas através da inibição da HMG-CoA redutase e da síntese de colesterol no fígado, ampliando o número de receptores de LDL hepáticos na superfície da célula, o que aumenta a absorção e o catabolismo do LDL. Indicações: para a redução de níveis elevados de colesterol total, LDL-colesterol, apolipoproteína $\mathrm{B}$ e triglicérides em pacientes com hipercolesterolemia primária e hiperlipidemia combinada (mista); para o tratamento de pacientes com níveis elevados de triglicérides séricos e de pacientes com disbetalipoproteinemia que não respondem de forma adequada à dieta; também é indicado para a redução do colesterol total e do LDL-colesterol em pacientes com hipercolesterolemia familiar homozigótica, quando a resposta à dieta e outras medidas não-farmacológicas forem inadequadas; em paciente com doença cardiovascular e/ou dislipidemia, está indicado na Síndrome Coronária Aguda, para a prevenção secundária do risco combinado de morte, infarto do miocárdio não-fatal, parada cardíaca e re-hospitalização de paciente com angina do peito (estudo MIRACL - Myocardial Ischemia Reduction with Aggressive Cholesterol Lowering). Contra-indicações: hipersensibilidade a qualquer componente de sua fórmula; doença hepática ativa ou elevações persistentes inesperadas das transaminases séricas que excedam em 3 vezes o limite superior da normalidade; durante a gravidez ou a lactação; mulheres em idade fértil que não estejam utilizando medidas contraceptivas eficazes. Advertências e precauções: os pacientes devem ser aconselhados a relatar imediatamente a ocorrência inexplicável de dor muscular, alterações da sensibilidade ou fraqueza muscular, principalmente se forem acompanhadas de malestar ou febre. Testes de função hepática devem ser realizados antes do início do tratamento e, periodicamente, durante o tratamento. Pacientes que desenvolverem quaisquer sinais ou sintomas sugestivos de danos hepáticos devem ser monitorados até que a anormalidade se resolva. Se um aumento de AST e ALT (TGO e TGP) maior que 3 vezes o limite superior da normalidade persistir, recomenda-se a redução da dose ou a descontinuação do tratamento. Citalor ${ }^{\circledR}$ deve ser utilizado com precaução por pacientes que consomem quantidades apreciáveis de álcool e/ou apresentam história de doença hepática. O tratamento deve ser descontinuado no caso de ocorrência de níveis consideravelmente elevados de CPK ou de diagnóstico ou suspeita de miopatia. O tratamento deve ser interrompido temporariamente ou descontinuado em qualquer paciente com uma condição séria e aguda sugestiva de miopatia ou que apresente um fator de risco que o predisponha ao desenvolvimento de insuficiência renal secundária à rabdomiólise. Interações medicamentosas: antiácidos (com hidróxido de magnésio e de alumínio), colestipol, digoxina, eritromicina/claritromicina, contraceptivos orais e inibidores da protease. Reações adversas: constipação, flatulência, dispepsia, dor abdominal, cefaléia, náusea, mialgia, astenia, diarréia e insônia. Posologia: as doses podem variar de 10 a $80 \mathrm{mg}$, em dose única diária, que pode ser administrada a qualquer hora do dia, com ou sem alimentos. As doses inicial e de manutenção devem ser individualizadas de acordo com os níveis basais de LDL-colesterol, o objetivo do tratamento e a resposta do paciente. Após o início do tratamento e/ou durante o ajuste de dose de atorvastatina, os níveis lipídicos devem ser analisados dentro de 2 a 4 semanas e a dose deve ser ajustada adequadamente. Hipercolesterolemia primária e hiperlipidemia combinada (mista): a maioria dos pacientes é controlada com $10 \mathrm{mg}$ de atorvastatina em dose única diária. Hipercolesterolemia familiar homozigótica: a maioria dos pacientes respondeu a $80 \mathrm{mg}$ de atorvastatina com uma redução maior que $15 \%$ no LDL-colesterol (18\%-45\%). A experiência no tratamento de pacientes pediátricos (com doses de atorvastatina de até $80 \mathrm{mg} / \mathrm{dia}$ ) é limitada. Superdosagem: o paciente deve receber tratamento sintomático e, conforme a necessidade, devem ser instituídas medidas de suporte. Devido à alta ligação às proteínas plasmáticas, a hemodiálise não deve aumentar a depuração da atorvastatina significantemente. Apresentações: comprimidos revestidos de $10 \mathrm{mg}, 20 \mathrm{mg}, 40 \mathrm{mg}$ e $80 \mathrm{mg}$ em embalagens com 30 unidades. USO ADULTO. VENDA SOB PRESCRIÇÃO MÉDICA. Para maiores informações, consulte a bula completa do produto (cit08a). Documentação científica e informações adicionais estão à disposição da classe médica mediante solicitação. Laboratórios Pfizer Ltda., Rua Alexandre Dumas, 1860 - Chácara Santo Antônio, São Paulo, SP - CEP 04717-904. Tel.: 0800-16-7575 - Internet: www.pfizer.com.br. Citalor ${ }^{\circledR}$ Reg. MS - 1. 0216.0062 